\title{
Immunohistochemical patterns in the differential diagnosis of rhinopharyngeal granulocytic sarcoma
}

\author{
ELENA CANTONE ${ }^{1}$, MICHELE CAVALIERE ${ }^{1}$, ANTONELLA MIRIAM DI LULLO ${ }^{1}$, \\ ELIA GUADAGNO ${ }^{2}$ and MAURIZIO IENGO ${ }^{1}$ \\ ${ }^{1}$ Department of Neuroscience, Ear, Nose and Throat Section; ${ }^{2}$ Department of Advanced Biomedical Sciences, \\ Pathology Section, 'Federico II' University of Naples, I-Naples 80100, Italy \\ Received February 21, 2016; Accepted May 16, 2016
}

DOI: $10.3892 / \mathrm{ol} .2016 .5009$

\begin{abstract}
Granulocytic sarcoma (GS) is a rare extramedullary manifestation of acute myeloid leukemia (AML). GS may develop simultaneously to AML or as a relapse of leukemia, particularly following allogeneic hematopoietic stem cell transplant. Subperiosteal bone, lymph nodes and skin are commonly involved, whereas rhinopharyngeal involvement is less common, with only 14 cases reported in the literature. Due to its rarity, rhinopharyngeal GS may lead to diagnostic pitfalls, particularly when it is poorly differentiated or is without concomitant marrow involvement. Thus, immunohistochemical findings play a key role in diagnosis. The current report describes a case of a 53-year-old female suffering from rhinopharyngeal GS and with a history of AML treated with chemotherapy and radiotherapy, focusing on the importance of the immunohistochemical pattern to assess the right diagnosis. Recent studies have demonstrated that the immunophenotype is of utmost importance for the diagnosis of GS. The high expression of myeloperoxidase (MPO) is common in GS; however, $\sim 30 \%$ of GSs do not contain MPO. Therefore, the presence of other markers is required to confirm the diagnosis of GS.
\end{abstract}

\section{Introduction}

Granulocytic sarcoma (GS) is a rare, extramedullary malignant neoplasm consisting of myeloid cells with different levels of maturation and occurring in anatomic sites other than the bone marrow or peripheral blood (1). It represents a distinct entity of AML (2).

The high expression of myeloperoxidase (MPO) makes these tumors green, hence their alternative name, 'chloroma' (from the Greek word 'chloros', meaning green). However,

Correspondence to: Dr Elena Cantone, Department of Neuroscience, Ear, Nose and Throat Section, 'Federico II' University of Naples, 5 Via Pansini, I-Naples 80100, Italy

E-mail: elenacantone@libero.it

Key words: granulocytic sarcoma, myeloid sarcoma, acute myeloid leukemia, rhinopharynx, tumors, immunohistochemistry 'sarcoma' is the most commonly used term, as $~ 30 \%$ of these tumors do not contain MPO, despite the fact that MPO together with cluster of differentiation (CD)117 represents the marker for myeloid differentiation (1).

GS may develop de novo (solitary, primary or non-leukemic GS; 8-20\%) (3), simultaneously to AML (2.5-9.1\%) (4), or as a relapse of leukemia, particularly following allogeneic hematopoietic stem cell transplant (AHSCT) $(4,5)$. The reason for this association is still unknown, but may be attributed to a pattern of graft-versus-leukemia surveillance or to the biology of high-risk AML treated with transplantation (5).

GS may affect patients of all ages (median age, 56 years; range, 1 month to 89 years), with a male:female ratio 1.2:1 (6). Commonly involved sites include subperiosteal bone, lymph nodes and skin. The prevalence of head and neck region involvement is $12-43 \%$ of cases (5), and orbit, skull and epidural spaces are the most frequently involved sites (7). Rare lesions have been described in the maxilla, soft palate, paranasal sinus, salivary gland, scalp and temporal bone, whereas only a few cases have been described with rhinopharyngeal involvement (4).

GS risk factors include specific chromosomal abnormalities $[\mathrm{t}(8 ; 21)$ and inv(16)], expression of cell-surface markers (CD56, CD2, CD4 and CD7), and M2, M4 and M5 leukemia subtypes of the French-American-British classification (4). Additional risk factors include poor nutritional status, cellular immune dysfunction, high presenting leukocyte count and decreased blast Auer rods (4).

Sinonasal congestion and/or hearing loss are the most common clinical manifestations of rhinopharyngeal GS (4).

The diagnosis of the rhinopharyngeal GS, particularly when it is poorly differentiated or without concomitant marrow involvement, is challenging (8), and it is not uncommon for GS to be misdiagnosed as lymphoma $(8,9)$. To improve the accuracy of diagnosis, immunohistochemical patterns play a key role. For instance, myeloid cells are reactive to antibodies against lysozyme, MPO and chloroacetate esterase. Furthermore, GS myeloblasts typically express myeloid-associated antigens, such as CD43, but are not reactive to lymphoid antigens. In addition, flow cytometry and cytogenetic analysis may aid in determining a definitive diagnosis (8).

GS is sensitive to focal irradiation and to systemic chemotherapy, similarly to AML $(7,10,11)$. Systemic treatment should 

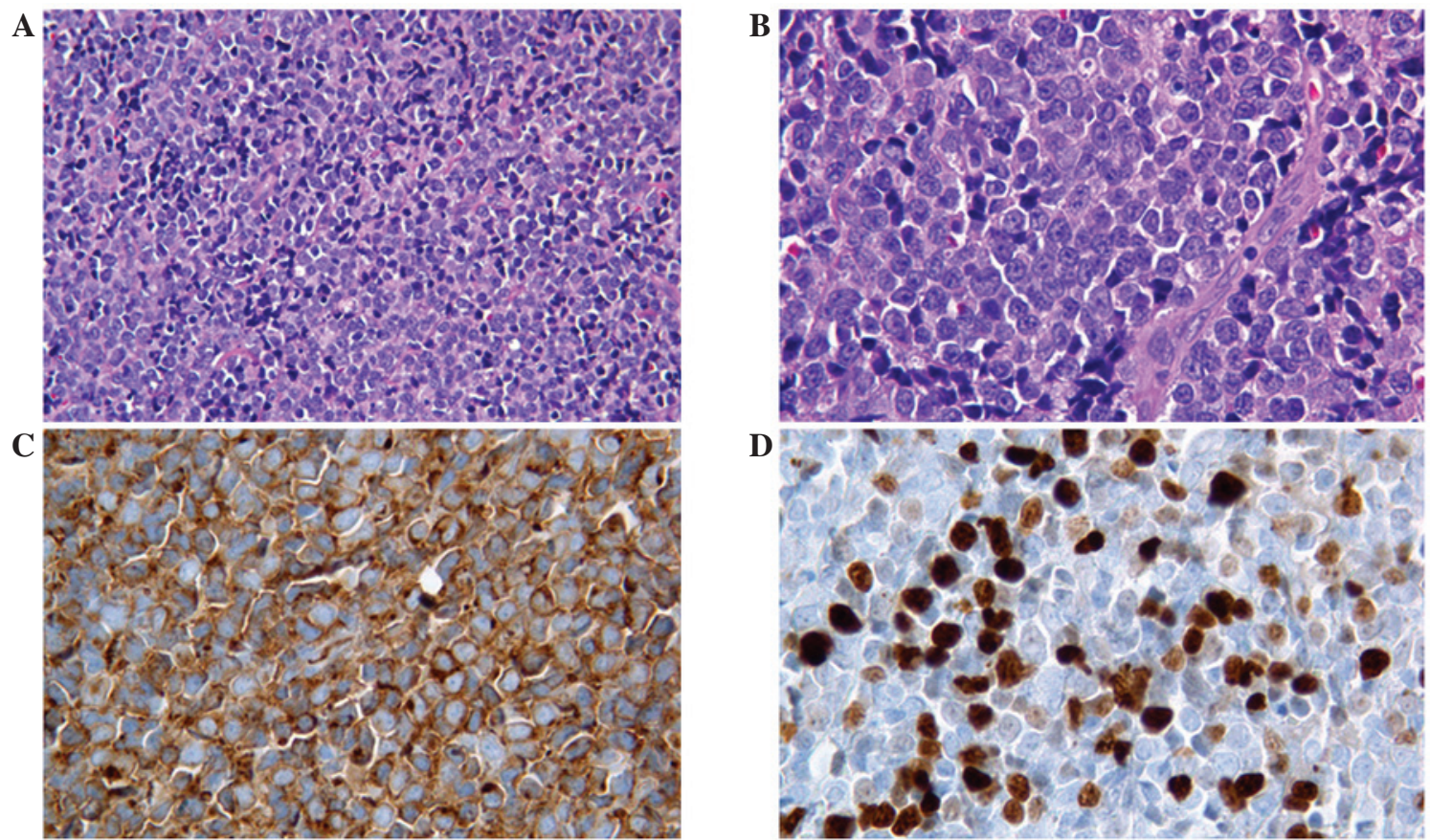

Figure 1. Histopathological analysis of biopsy specimen. (A and B) The neoplastic cell population was composed of small and medium sized elements, characterized by an inconspicuous eosinophilic cytoplasm and a nucleus with an irregular nuclear membrane; occasionally a small nucleolus was observed [hematoxylin and eosin staining; magnification, (A) x20 and (B) x40]. (C) Neoplastic cells were strongly immunoreactive for cluster of differentiation 43. (D) A high proliferative index was observed (Ki-67,40\%).

always be considered due to the high rate of recurrence and progression to AML (11). Surgery may be a therapeutic option only for tumors, which cause organ dysfunction (11). The role of radiotherapy and AHSCT as a consolidation regimen remains to be clearly established $(10,11)$.

GS has an unfavorable prognosis. Its course is rapid with a high mortality rate, particularly when associated with AML, whereas patients without evidence of leukemia have a better prognosis (9); cases initially diagnosed as solitary GS without evidence of leukemia and treated with systemic chemotherapy have a more favorable prognosis (9).

The current study reports the case of a 53-year-old woman who presented with a rhinopharyngeal mass. The mass was diagnosed as an isolated extramedullary GS as a relapse of AML, which had been treated 7 years earlier with chemotherapy and AHSCT and was followed by complete remission. In addition, a review of the literature is reported, along with the examination of immunohistochemical features as a tool for the differential diagnosis of GS compared to other rare tumors of the rhinopharynx.

\section{Case report}

A 53-year-old female non-smoker presented to the Ear, Nose and Throat Unit of 'Federico II' University of Naples (Naples, Italy) complaining of left otalgy, hearing loss and nasal obstruction for $\sim 5$ months. The patient had a history of AML, which was in complete remission following high-dose chemotherapy and AHSCT, administered 7 years earlier.

A nasal endoscopy revealed a left rhinopharyngeal peritubaric mass obstructing the left Eustachian tube. The audiological evaluation revealed left conductive hearing loss due to an ipsilateral middle ear effusion. No lateral cervical palpable lymph nodes were found. Magnetic resonance imaging and positron emission tomography-computed tomography (CT) examinations revealed a mass of the rhinopharynx $(6 \times 3.5 \mathrm{~cm}$; standardized uptake value, 5.7) without bone erosion.

Laboratory studies, including a normal complete blood count, unremarkable serum chemistry and normal liver enzyme levels, did not reveal any alteration. No evidence of increased blast cell count was observed in the bone marrow aspiration sample.

The specimen was formalin fixed and paraffin embedded. Immunohistochemistry was performed using the avidin biotin complex as a visualization system and 3,3'-diaminobenzidine as chromogen for the reaction, with pre-diluted antibodies (dilution, 1:100) against B-cell lymphoma-2 (Bcl-2; 790-4604, clone SP66; rabbit; Ventana Medical Systems, Inc., Tucson, AZ, USA), CD3 (790-4341; clone 2GV6; rabbit; Ventana Medical Systems, Inc.), CD5 (790-4451; clone SP19; rabbit; Ventana Medical Systems, Inc.), CD10 (790-4506; clone SP67; rabbit; Ventana Medical Systems, Inc.), CD20 (760-2531; clone L26; mouse; Ventana Medical Systems, Inc.), CD34 (7902927; clone QBEnd/10; mouse; Ventana Medical Systems, Inc.), CD43 (760-2511; clone L60; mouse; Ventana Medical Systems, Inc.), CD56 (790-4465; clone 123C3; mouse; Ventana Medical Systems, Inc.), CD99 (790-4452; clone O13; mouse; Ventana Medical Systems, Inc.), CD117 (790-2951; clone 9.7; rabbit; Ventana Medical Systems, Inc.), Ki67 (M724029; clone MIB1; mouse; Dako, Glostrup, Denmark) and MPO (7602659; rabbit polyclonal; Ventana Medical Systems, Inc.). The patient underwent biopsy of the rhinopharyngeal lesion, which revealed neoplastic proliferation of medium- and small-sized cells. These cells exhibited inconspicuous cytoplasm, nuclei with irregular membranes, occasionally a small nucleolus, diffuse karyorrhexis and high mitotic activity (Fig. 1). 


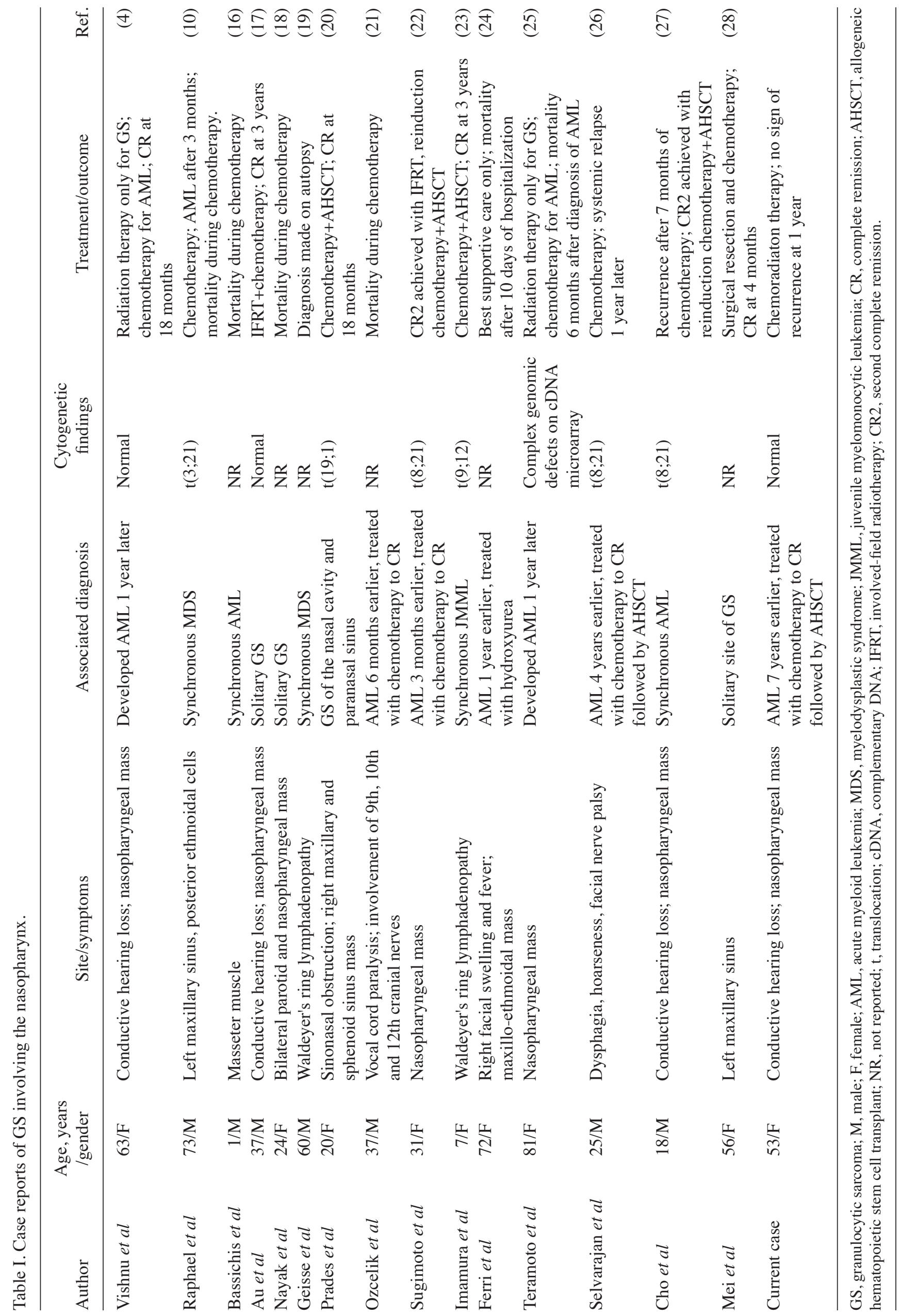


The immunohistochemical evaluation revealed strong reactivity for $\mathrm{CD} 43, \mathrm{CD} 34$ and $\mathrm{CD} 99$, whereas $\mathrm{CD} 20, \mathrm{CD} 3$, CD5, CD10, Bcl-2, MPO, CD117 and CD56 were all negative. The Ki-67 proliferative index was $\sim 40 \%$. Fluorescence in situ hybridization (FISH) analysis was performed on $4-\mu \mathrm{m}$-thick formalin-fixed, paraffin-embedded (FFPE) tissue sections, using the Vysis LSI Dual Color probe (Abbott Molecular, Inc., Des Plaines, IL, USA) specific for runt-related transcription factor 1 (RUNX1) labeled with Spectrum Green and for RUNX1T1 labeled with Spectrum Orange. The slides were hybridized overnight according to the manufacturer's protocol. Image analysis was then conducted. The FISH analysis of FFPE tumor slides did not identify either the $t(8 ; 21)$ translocation or $M L L$ gene dissociation.

The patient's medical history and immunophenotype suggested the presence of a poorly differentiated extramedullary GS of the rhinopharynx as a relapse of AML, without bone marrow disease.

The patient was treated with conventional induction AML therapy: Combined idarubicin $\left(12 \mathrm{mg} / \mathrm{m}^{2} /\right.$ day, days $\left.1-2\right)$ and cytarabine $\left(200 \mathrm{mg} / \mathrm{m}^{2} /\right.$ day, days $\left.1-7\right)$, followed by one course of consolidation therapy with idarubicin $\left(12 \mathrm{mg} / \mathrm{m}^{2}\right.$, day 1$)$ and cytarabine $\left(1 \mathrm{~g} / \mathrm{m}^{2} / 12 \mathrm{~h}\right.$, days $\left.1-5\right)$, as well as radiation treatment (1,500 cGy in 5 fractions).

A CT scan performed at 1 month after chemoradiotherapy revealed complete resolution of the rhinopharyngeal mass. At the 3-year follow-up, the patient was asymptomatic and without signs of recurrence. At present, the patient is under a regular surveillance protocol; she is closely monitored through a multidisciplinary 'short time' follow-up protocol, consisting of nasal endoscopy and blood studies every 3 months.

Written informed consent was obtained from the patient for publication of this case report and the accompanying images.

\section{Discussion}

GS, also known as 'chloroma' or 'extramedullary myeloblastoma', is a rare solid tumor consisting of primitive precursors of the granulocytic series of white blood cells, which include myeloblasts, promyelocytes, and myelocytes (1). Rhinopharyngeal localization of GS is extremely rare; only a few cases have been previously reported in the literature (4), and the present study reports the 15 th case (Table I).

GS has a high rate of misdiagnosis $(46 \%)(12,13)$, and its differential diagnosis may include non-Hodgkin's lymphoma (lymphoblastic, Burkitt and diffuse large B-cell lymphomas), lymphoblastic leukemia, melanoma, Ewing's sarcoma, primitive neuroectodermal tumor, rhabdomyosarcoma, neuroblastoma, medulloblastoma, undifferentiated carcinoma, blastic plasmacytoid dendritic cell neoplasm, extramedullary hematopoiesis (13) and small undifferentiated round cell tumors $(6,14)$.

Previous studies have demonstrated that immunophenotype is of utmost importance in determining the diagnosis of GS (3-5). In particular, the literature has focused on the CD13 and CD68 markers for granular monocytic and macrophagic cells, MPO and CD117 markers for myeloid differentiation, lysozyme marker for monocytic lineage, CD43 marker for myeloid cells as well as T cells and B precursors, and CD34 and terminal deoxynucleotidyl transferase (TdT) markers for immature cells (10). Immunohistochemical detection of intracellular MPO, a major constituent of primary granules of neutrophilic myeloid cells, confirms a diagnosis of GS. However, while MPO is expressed in the majority of GSs, some minimally differentiated and monocytic GSs do not express it (8).

CD68-KP1 is the most commonly expressed marker, followed by MPO, CD117, CD99, CD68/PG M1, lysozyme, CD34, TdT, CD56, CD61/linker of activated T lymphocyte/factor VIII-related antigen, CD30, glycophorin A and CD4 (3). Rarely, aberrant antigenic expression is observed (such as cytokeratins, B- or T-cell markers) (3).

In the current case, immunohistochemical evaluation revealed strong reactivity for CD43, CD34 and CD99, whereas MPO was negative. In particular, the presence of CD99 made it difficult to differentiate GS from other CD99-positive round cell tumors (14), whereas the positivity for CD34 (15) and the negativity for MPO indicated the presence of a mass of immature cells. In the present case, the clinical history of previous AML was the key to the specific diagnosis of GS.

From a therapeutic perspective, data from the literature suggest that GSs are extremely sensitive to focal irradiation or chemotherapy; however, their role is not well defined $(13,16-28)$. The optimal treatment for the GS-AML association remains uncertain. However, high-dose chemotherapy and stem cell transplantation may be the treatment of choice (6).

The risk of metachronous AML in non-leukemic patients with GS is very high, with a median delay of 5 months; the majority of patients may develop AML within 1 year. Therefore, early intensive (induction/intensification) chemotherapy similar to that used to treat AML must be administered, even in GS patients without AML upon initial diagnosis (6).

Byrd et al stated that $97 \%$ of all primary GS patients who did not receive systemic chemotherapy developed AML (12). Furthermore, $66 \%$ of patients who received chemotherapy for the primary GS did not develop AML, suggesting that early systemic therapy is helpful in preventing AML, which increases the overall survival time (6).

Literature data demonstrated that clinical behavior and response to therapy are not influenced by any of the following factors: Age, gender, anatomic site, de novo presentation, histotype, phenotype or cytogenetic findings (6).

In the current case, given the previous history of AML and according to data in the literature, the patient was treated with conventional induction AML therapy, followed by radiotherapy. This choice, so far, has proved to be a successful strategy, since the patient has shown no signs of relapse after 3-year follow-up.

In conclusion, the current study highlights certain interesting features of GS. Firstly, the negative reactivity for MPO in the current case suggested the diagnosis of a poorly differentiated GS with a poor prognosis. Thus, the patient was assigned to a multidisciplinary protocol of close follow-up for the high risk of relapse. Secondly, since the rhinopharynx is involved in a variety of malignant neoplasms, immunohistochemistry is required for the diagnosis of GS, particularly for the undifferentiated forms, as in the present case. Indeed, it is not uncommon for GS to be misdiagnosed as lymphoma. Finally, a combination of detailed clinical, radiographic and serological work-ups, in association with a thorough histological assessment, is essential to establish the correct diagnosis (29). 


\section{References}

1. Guermazi A, Feger C, Rousselot P, Merad M, Benchaib N, Bourrie P, Mariette X, Frija J and de Kerviler E: Granulocytic sarcoma (chloroma): Imaging findings in adults and children AJR Am J Roentgenol 178: 319-325, 2002.

2. Vardiman JW, Thiele J, Arber DA, Brunning RD, Borowitz MJ, Porwit A, Harris NL, Le Beau MM, Hellström-Lindberg E, Tefferi A and Bloomfield CD: The 2008 revision of the World Health Organization (WHO) classification of myeloid neoplasms and acute leukemia: Rationale and important changes. Blood 114: 937-951, 2009.

3. Campidelli C, Agostinelli C, Stitson R and Pileri SA: Myeloid sarcoma: Extramedullary manifestation of myeloid disorders. Am J Clin Pathol 132: 426-437, 2009.

4. Vishnu P, Chuda RR, Hwang DG and Aboulafia DM: Isolated granulocytic sarcoma of the nasopharynx: A case report and review of the literature. Int Med Case Rep J 7: 1-6, 2013.

5. Alrumaih R, Saleem M, Velagapudi S and Dababo MA: Latera pharyngeal wall myeloid sarcoma as a relapse of acute biphenotypic leukemia: A case report and review of the literature. J Med Case Rep 7: 292, 2013.

6. Messager M, Amielh D, Chevallier C and Mariette C: Isolated granulocytic sarcoma of the pancreas: A tricky diagnostic for primary pancreatic extramedullary acute myeloid leukemia World J Surg Oncol 10: 13, 2012.

7. Noh BW, Park SW, Chun JE, Kim JH, Kim HJ and Lim MK Granulocytic sarcoma in the head and neck: CT and MR imaging findings. Clin Exp Otorhinolaryngol 2: 66-71, 2009.

8. Papamanthos MK, Kolokotronis AE, Skulakis HE, Fericean AA Zorba MT and Matiakis AT: Acute myeloid leukaemia diagnosed by intra-oral myeloid sarcoma. A Case Report. Head Neck Pathol 4: 132-135, 2010.

9. Hwang JI and Kim TY: Primary granulocytic sarcoma of the face. Ann Dermatol 23 (Suppl 2): S214-S217, 2011.

10. Raphael J, Valent A, Hanna C, Auger N, Casiraghi O, Ribrag V, De Botton S and Saada V: Myeloid Sarcoma of the nasopharynx mimicking an aggressive lymphoma. Head Neck Pathol 8: 234-238, 2014.

11. Yilmaz AF, Saydam G, Sahin F and Baran Y: Granulocytic sarcoma: A systematic review. Am J Blood Res 3: 265-270, 2013.

12. Byrd JC, Edenfield WJ, Shields DJ and Dawson NA: Extramedullary myeloid cell tumors in acute nonlymphocytic leukemia: A clinical review. J Clin Oncol 13: 1800-1816, 1995.

13. Vachhani $\mathrm{P}$ and Bose P: Isolated gastric myeloid sarcoma: A case report and review of the literature. Case Rep Hematol 2014: 541807,2014

14. Casiraghi $\mathrm{O}$ and Lefèvre $\mathrm{M}$ : Undifferentiated malignant round cell tumors of the sinonasal tract and nasopharynx. Ann Pathol 29: 296-312, 2009 (In French).

15. Cheng Y, Jia M, Chen Y,Zhao H, Luo Z and Tang Y: Re-evaluation of various molecular targets located on CD34+CD38-Linleukemia stem cells and other cell subsets in pediatric acute myeloid leukemia. Oncol Lett 11: 891-897, 2016.
16. Bassichis B, McClay J and Wiatrak B: Chloroma of the masseteric muscle. Int J Pediatr Otorhinolaryngol 53: 57-61, 2000.

17. Au WY, Kwong YL, Ho WK and Shek TW: Primary granulocytic sarcoma of the nasopharynx. Am J Hematol 67: 273-274, 2001.

18. Nayak DR, Balakrishnan R, Raj G, Pillai S, Rao L and Manohar C: Granulocytic sarcoma of the head and neck: A case report. Am J Otolaryngol 22: 80-83, 2001.

19. Geisse M, Mall G, Fritze D and Gartenschläger M: Granulocytic sarcoma of the tonsils associated with myelodysplastic syndrome. Dtsch Med Wochenschr 127: 2673-2676, 2002 (In German).

20. Prades JM, Alaani A, Mosnier JF, Dumollard JM and Martin C: Granulocytic sarcoma of the nasal cavity. Rhinology 40: 159-161, 2002.

21. Ozçelik T, Ali R, Ozkalemkaş F, Ozkocaman V, Coşkun H, Erişen L and Filiz G: A case of granulocytic sarcoma during complete remission of acute myeloid leukemia with multiple masses involving the larynx and nasopharynx. Kulak Burun Bogaz Ihtis Derg 11: 183-188, 2003.

22. Sugimoto Y, Nishii K, Sakakura M, Araki H, Usui E, Lorenzo VF, Hoshino N, Miyashita H, Ohishi K, Katayama N and Shiku H: Acute myeloid leukemia with $\mathrm{t}(8 ; 21)(\mathrm{q} 22 ; \mathrm{q} 22)$ manifesting as granulocytic sarcomas in the rhinopharynx and external acoustic meatus at relapse after high-dose cytarabine: Case report and review of the literature. Hematol J 5: 84-89, 2004.

23. Imamura $\mathrm{T}$, Matsuo $\mathrm{S}$, Yoshihara $\mathrm{T}$, Chiyonobu $\mathrm{T}$, Mori $\mathrm{K}$, Ishida H, Nishimura Y, Kasubuchi Y, Naya M, Morimoto A, et al: Granulocytic sarcoma presenting with severe adenopathy (cervical lymph nodes, tonsils, and adenoids) in a child with juvenile myelomonocytic leukemia and successful treatment with allogeneic bone marrow transplantation. Int J Hematol 80: 186-189, 2004

24. Ferri E, Minotto C, Ianniello F, Cavaleri S, Armato E and Capuzzo P: Maxillo-ethmoidal chloroma in acute myeloid leukaemia: Case report. Acta Otorhinolaryngol Ital 25: 195-199, 2005.

25. Teramoto H, Miwa H, Patel V, Letwin N, Castellone MD, Imai N, Shikami M, Imamura A, Gutkind JS, Nitta M and Lee NH: Gene expression changes in a patient presenting nonleukaemic nasal granulocytic sarcoma to acute myelogenous leukaemia using $40 \mathrm{~K}$ cDNA microarray. Clin Lab Haematol 28: 262-266, 2006.

26. Selvarajan S, Subramanian S, Thulkar S and Kumar L: Granulocytic sarcoma of nasopharynx with perineural spread along the trigeminal nerve. Neurol India 56: 210-212, 2008.

27. Cho SF, Liu YC, Tsai HJ and Lin SF: Myeloid sarcoma mimicking nasopharyngeal carcinoma. J Clin Oncol 29: e706-e708, 2011.

28. Mei KD, Lin YS and Chang SL: Myeloid sarcoma of the cheek and the maxillary sinus regions. J Chin Med Assoc 76: 235-238, 2013.

29. Cantone E, Marino A, Ferranti I and Iengo M: Nonallergic rhinitis in the elderly: A reliable and safe therapeutic approach. ORL J Otorhinolaryngol Relat Spec 77: 117-22, 2015. 